\title{
Citral Delays Postharvest Senescence of Kiwifruit by Enhancing Antioxidant Capacity under Cold Storage
}

\author{
Ling Wei, ${ }^{1,2}$ Chuying Chen $\mathbb{D}^{1},{ }^{1}$ Chunpeng Wan $\mathbb{D}^{1},{ }^{1}$ Ming Chen, ${ }^{1}$ and Jinyin Chen $\mathbb{D}^{1,3}$ \\ ${ }^{1}$ Jiangxi Key Laboratory for Postharvest Technology and Nondestructive Testing of Fruits \& Vegetables, \\ Collaborative Innovation Center of Postharvest Key Technology and Quality Safety of Fruits \& Vegetables in Jiangxi Province, \\ College of Agronomy, Jiangxi Agricultural University, Nanchang 330045, China \\ ${ }^{2}$ Department of Chemistry, College of Science, Jiangxi Agricultural University, Nanchang 330045, China \\ ${ }^{3}$ College of Materials and Chemical Engineering, Pingxiang University, Pingxiang 337055, China
}

Correspondence should be addressed to Chuying Chen; cy.chen@jxau.edu.cn and Jinyin Chen; jinyinchen@126.com

Received 3 December 2020; Revised 27 January 2021; Accepted 5 February 2021; Published 17 February 2021

Academic Editor: Laura Arru

Copyright (c) 2021 Ling Wei et al. This is an open access article distributed under the Creative Commons Attribution License, which permits unrestricted use, distribution, and reproduction in any medium, provided the original work is properly cited.

Citral is an aliphatic aldehyde extracted from citrus essential oil. The aim of the study was to examine how citral treatment affects the weight loss, firmness, respiration, and ripening index, as well as the antioxidant capacity of kiwifruit (Actinidia chinensis $c v$. 'Jinkui'). The citral treatment was seen to reduce the weight loss, softening, and fruit respiration compared to control fruits. Citral treatment also had an inhibitory effect on ripening index, $\mathrm{O}_{2}{ }^{--}$production rate, and malondialdehyde (MDA) accumulation. The degradations of ascorbic acid (AsA) content, total flavonoids content (TFC), and total phenolics content (TPC) were also suppressed by citral. In contrast, citral treatment induces the activation of antioxidant enzyme system such as superoxide dismutase (SOD), peroxidase (POD), and catalase (CAT). Collectively, the results indicated that citral treatment delays postharvest senescence and prolongs storage life by enhancing antioxidant capacity in harvested kiwifruits. These findings suggest that citral has the potential to be used as a promising natural preservative for the extension of postharvest quality in harvested kiwifruit.

\section{Introduction}

Kiwifruit (Actinidia deliciosa, Actinidiaceae) is quite popular because of its taste and having high vitamin $\mathrm{C}$ and antioxidant capacities [1]. Kiwifruit is highly perishable and susceptible to pathogenic fungi causing significant economic losses due to the rotting of fruits during storage $[2,3]$. Till date, various postharvest approaches such as edible coatings (alginate oligosaccharide, chitosan, and lacquer wax) $[2,4,5], 24$-epibrassinolide [6], oxalic acid [7, 8], aminoethoxyvinylglycine [9], methyl jasmonate [10], hydrogen sulfide [11, 12], and ozone treatment [13] have been implied and found to prolong the postharvest storability of kiwifruit. Postharvest application of natural phytochemicals, particularly plant essential oils, has been proved to be effective in controlling diseases and preservation of fruit quality $[14,15]$.

Recently, essential oils (EO) and their various constituents are used as natural fungicides for controlling postharvest fruits diseases and have been extensively studied. Essential oils from Artemisia herba-alba, Mentha pulegium, and Syzygium aromaticum had certain antimicrobial effects on Penicillium expansum population and blue mold development of apples [15]. Moreover, cinnamon essential oil displayed a strong antifungal inhibition on Colletotrichum acutatum of 'Hongyang' kiwifruit [16], and cassia oil has been used against Alternaria alternata (black spot rot) in tomatoes [17]. It is also shown that some essential oils could enhance antioxidant capacities of many horticultural products such as strawberries and blueberries $[18,19]$. Citral, a terpenoid isolated from Litsea cubeba and Cymbopogon citratus (lemongrass), is widely used for food, beverages, and cosmetics industry [20]. Recently, citral has been reported to have significant antifungal effects on postharvest pathogens such as P. italicum, $P$. digitatum, and Geotrichum citri aurantii $[21,22]$, which effectively enhances the antioxidant capacities and disease resistance in some fruits $[23,24]$. In 
addition to these, citral has been used a promising plantderived pesticide, which seems to be a surprising application to protect horticultural products from fungal attacks $[21,24]$. Therefore, it remains a mighty possibility that citral could be used commercially for controlling postharvest fungal decay and enhancing disease resistance of horticultural products.

To develop a postharvest preservation using more applicable form of citral for kiwifruit, the effects of citral on the postharvest quality and on the response of reactive oxygen species (ROS) and antioxidant capacity shall also be studied in the present study.

\section{Materials and Methods}

2.1. Materials and Treatment. Healthy "Jinkui" kiwifruits (Actinidia chinensis) were harvested from a commercial orchard in Fengxin County (Jiangxi Province, China) with a commercial maturity [mean flesh firmness: $7.19 \mathrm{~N}$; soluble solids content (SSC): $7.61 \pm 0.16 \%$ ]. The picked fruits with uniform size, color, and shape, free from any mechanical damage or pests were randomly divided into 39 groups comprising 10 fruits each. Eighteen groups were dipped in $0.6 \mu \mathrm{LmL}^{-1}$ citral (prepared in $0.1 \%$ Tween 80 solution) for $15 \mathrm{~min}$, and another eighteen groups immersed in $0.1 \%$ Tween 80 solution only, to serve as the controls. The citral concentration of $0.6 \mu \mathrm{LmL}^{-1}$ was chosen based on the evaluation in a preliminary experiment using $0,0.2,0.4,0.6$, and $0.8 \mu \mathrm{LmL}^{-1}$ citral solutions (data not shown). The remaining three groups were also sampled before treatment to determine physicochemical quality parameters at harvest time (0 day). Both citral-treated and control kiwifruits were air-dried (at $20 \pm 1^{\circ} \mathrm{C}$ and $90 \pm 5 \%$ relative humidity), packed in lowdensity polyethylene bags $(39 \times 30 \mathrm{~cm}, 0.03 \mathrm{~mm}$ thickness), and then stored at $0-1^{\circ} \mathrm{C}$ and $90 \pm 5 \% \mathrm{RH}$ for $90 \mathrm{~d}$. Three replicates of 30 fruits for each treatment were taken at intervals of $15,30,45,60,75$, and $90 \mathrm{~d}$ and sampled the flesh tissue for determination of flesh firmness, quality parameters, membrane permeability, and antioxidant enzyme activities.

\subsection{Evaluation of Weight Loss, Fresh Firmness, Total Soluble} Solids (TSS) and Titratable Acid (TA) Content, and Ripening Index. The weight of the same 10 kiwifruits from the control and citral-treated groups was measured immediately after days 0 and at the different sampling times $(15,30,45,60,75$, and $90 \mathrm{~d}$ ). Weight loss is defined as the ratio of final sample weights to the harvested sample weights and expressed as percentage (\%).

Fresh firmness of kiwifruit was evaluated on two opposite positions around the equator of 10 fruits (skin removed) using a texture analyzer (TAXT Plus, Stable Micro Systems, Surrey, UK) equipped with a $2 \mathrm{~mm}$ diameter probe and expressed as $\mathrm{N}$.

The SSC in kiwifruit juice was recorded on a digital Brixmeter (RA-250WE, KYOTO, Tokyo, Japan) and specified in ${ }^{\circ}$ Brix. TA content was analyzed by the titration with $0.1 \mathrm{M}$ $\mathrm{NaOH}$ and expressed in terms of the percentage of citric acid following the method described by Horak et al. [25].
Ripening index was calculated by simply taking the ratio of SSC and TA (SSC:TA).

2.3. Respiration Rate, $\mathrm{O}_{2}{ }^{--}$Production Rate, and Malondialdehyde (MDA) Content. The respiration rate was determined according to a method described by $\mathrm{Hu}$ et al. [5]. The respiration rate was analyzed by an infrared $\mathrm{CO}_{2}$ fruit breathing instrument (GHX-3051H, Shanghai, China) and recorded as $\mathrm{mg} \mathrm{CO}_{2} \mathrm{~kg}^{-1} \mathrm{~h}^{-1}$.

For $\mathrm{O}_{2}{ }^{--}$production rate assay, $2.0 \mathrm{~g}$ frozen tissue powder was homogenized with $5 \mathrm{~mL}$ ice-cooled $50 \mathrm{mM}$ phosphate buffer (pH 7.8) containing 1 mM EDTA, $0.3 \%(\mathrm{v} /$ v) Triton X-100, and 2\% (w/v) polyvinyl pyrrolidone (PVP) and then centrifuged at $10000 \times \mathrm{g}$ for $20 \mathrm{~min}$ at $4^{\circ} \mathrm{C} .1 \mathrm{~mL}$ of the supernatant was mixed with $1 \mathrm{~mL}$ of $50 \mathrm{mM}$ phosphate buffer $(\mathrm{pH} 7.8)$ and $1 \mathrm{~mL}$ of $1 \mathrm{mM}$ hydroxylammonium hydrochloride and incubated for $1 \mathrm{~h}$ at $25^{\circ} \mathrm{C} .2 \mathrm{~mL}$ of the mixed solution comprised of $17 \mathrm{mM}$ 4-aminobenzenesulfonic acid and $7 \mathrm{mM}$ naphthylamine $(1: 1)$ was then added into the mixture, followed by incubation for $20 \mathrm{~min}$ at $25^{\circ} \mathrm{C}$. The absorbance of the mixture was recorded at $530 \mathrm{~nm}$. The unit of $\mathrm{O}_{2}{ }^{--}$production rate was expressed as nmol $\mathrm{g}^{-1} \min ^{-1}$.

MDA content was measured using the thiobarbituric acid colorimetric method according to the procedure of Nie et al. [26]. Frozen flesh tissue was milled to a powder with liquid nitrogen, and $1.0 \mathrm{~g}$ of powder was homogenized in $5 \mathrm{~mL} 10 \%(\mathrm{~m} / \mathrm{v})$ trichloroacetic acid (TCA) and then centrifuged at $10000 \times \mathrm{g}$ for $15 \mathrm{~min} .2 \mathrm{~mL}$ of the obtained supernatant was mixed with $2 \mathrm{~mL}$ of $0.67 \%$ thiobarbituric acid, followed by boiling water bath for $15 \mathrm{~min}$. The mixture was then cooled and the absorbance of the supernatant was determined at 450, 532, and $600 \mathrm{~nm}$. Each treatment was conducted in triplicate.

\subsection{Assays for Ascorbic Acid (AsA) Content, Total Phenolics} Content (TPC), and Total Flavonoids Content (TFC). Ascorbic acid (AsA) content of kiwifruit flesh was acquired by the titration with a standard solution of 2,6-dichlorophenol indophenol [26] and expressed in terms of milligram on a fresh weight basis $\left(\mathrm{g} \mathrm{kg}^{-1}\right)$.

The levels of TPC and TFC were measured following the method described by Jiang et al. [27]. Both TPC and TFC were represented as milligram of gallic acid and rutin equivalent per gram of frozen sample $\left(\mathrm{mg} \mathrm{kg}^{-1}\right)$, respectively.

2.5. Assay of 2,2-Diphenyl-1-picrylhydrazyl (DPPH) Scavenging Capacity and Ferric Reducing Antioxidant Power (FRAP). The DPPH scavenging capacity was carried out according to a previous method described by Horak et al. [25]. $100 \mu \mathrm{L}$ of extracted juice was added to $1.9 \mathrm{~mL}$ of $0.1 \mathrm{mM}$ DPPH solution (dissolved in ethanol) and then left in the dark at $25^{\circ} \mathrm{C}$ for $30 \mathrm{~min}$. The absorbance of the tested sample was monitored at $515 \mathrm{~nm}$. DPPH free radical scavenging capacity was calculated and expressed as a percentage using the following equation: 


$$
\text { Scavenging capacity }(\%)=\frac{A_{0}-A_{t}}{A_{0}} \times 100,
$$

where $A_{0}$ and $A_{t}$ are the absorbance of the control (without extracted juice) and kiwifruit juice added after $30 \mathrm{~min}$ of reaction incubation, respectively. The unit of DPPH scavenging capacity was expressed as \%.

The FRAP assay was carried out according to the method of Benzie and Strain [28] with a slight modification. $0.5 \mathrm{~mL}$ of extracted juice was added to $3 \mathrm{~mL}$ of fresh FRAP reagent [0.3 M acetate buffer ( $\mathrm{pH} 3.6$ ), $10 \mathrm{mM} \mathrm{2,} \mathrm{4,} \mathrm{6-tripyridyl-s-}$ triazine (dissolved in $40 \mathrm{mM} \mathrm{HCl}$ ), and $20 \mathrm{mM} \mathrm{FeCl}_{3} 6 \mathrm{H}_{2} \mathrm{O}$ $(10: 1: 1, \mathrm{v} / \mathrm{v} / \mathrm{v})]$ and then left in the dark at $37^{\circ} \mathrm{C}$ for $30 \mathrm{~min}$. The absorbance of the mixture was determined at $700 \mathrm{~nm}$ using distilled $\mathrm{H}_{2} \mathrm{O}$ as the blank solution.

2.6. Measurement of Antioxidant Enzyme Activities. Fresh tissue $(2.0 \mathrm{~g})$ from 10 fruits was homogenized with $8 \mathrm{~mL}$ of ice-cold $50 \mathrm{mM}$ phosphate buffer solution ( $\mathrm{pH} 7.0)$ containing $2 \%$ polyvinyl pyrrolidone and $5 \mathrm{mM}$ dithiothreitol and then centrifuged at $12,000 \times \mathrm{g}$ for $20 \mathrm{~min}$ at $4^{\circ} \mathrm{C}$. The obtained supernatant was used to determine the activity of the antioxidant enzymes.

Superoxide dismutase (SOD, EC 1.15.1.1) activity was measured using a SOD test kit (No:A001-1-2; Nanjing Jiancheng Bioengineering Institute, Nanjing, China) with the hydroxylamine method. The absorbance of the tested sample was monitored at $550 \mathrm{~nm}$. The quantity of enzyme that controlled $50 \%$ of nitroblue tetrazolium (NBT) photoreduction per hour was defined as one unit of SOD activity.

Catalase (CAT, EC 1.11.1.6) activity was determined by measuring the decline of absorbance at $240 \mathrm{~nm}$ (Shimadzu UV-2600, Tokyo, Japan) due to the decomposition of $\mathrm{H}_{2} \mathrm{O}_{2}$. The collected supernatant $(200 \mu \mathrm{L})$ was mixed with $2.8 \mathrm{~mL}$ of $20 \mathrm{mM} \mathrm{H}_{2} \mathrm{O}_{2}$ (prepared in $50 \mathrm{mM}$ phosphate buffer solution). The quantity of CAT that changed the absorbance of the reaction solution by 0.01 at $240 \mathrm{~nm}$ per minute was defined as one unit of CAT activity.

Peroxidase (POD, EC 1.11.1.7) activity was assayed using the guaiacol method reported by our previous studies $[3,26]$. The reaction system consisted of $50 \mu \mathrm{L}$ of enzyme solution, $3.0 \mathrm{~mL}$ of $25 \mathrm{mM}$ guaiacol (prepared in $50 \mathrm{mM}$ phosphate buffer solution), and $200 \mu \mathrm{L}$ of $0.5 \mathrm{mM} \mathrm{H}_{2} \mathrm{O}_{2}$. The increase in absorbance at $470 \mathrm{~nm}$ was determined at $25^{\circ} \mathrm{C}$ for $5 \mathrm{~min}$. The quantity of POD that changed the absorbance of the reaction solution by 1 at $470 \mathrm{~nm}$ per minute was defined as one unit of POD activity.

2.7. Statistical Analysis. All data were described as the means of three replicated samples \pm standard error (S.E.), and analyses were carried out by using SPSS version 20.0 (SPSS Inc., Chicago, IL, USA). $P$ value less than $0.05(p<0.05)$ was considered as significant. Origin version 8.5 (Microcal Software, Northampton, MA) was applied to create figures.

\section{Results}

3.1. Change of Fruit Weight Loss and Firmness after Citral Treatment. Fruit weight loss in both control and citraltreated fruits depicted a clear increase during storage (Figure 1). However, the weight loss in the fruit treated with citral maintained a low level compared with the control fruits. By the end of cold storage period of $90 \mathrm{~d}$, the weight loss in the citral-treated kiwifruit was lower $(18.9 \%, p<0.05)$ than that in the control fruits.

Flesh firmness in the control and citral-treated kiwifruit decreased throughout the storage period (Figure 2). Further comparison indicated that there was a higher level of flesh firmness in the citral-treated kiwifruit than that in the control kiwifruit under storage, with a remarkable $(p<0.05)$ difference during the entire storage period.

\subsection{Effects of Citral Treatment on Postharvest Senescence} Related Parameters of Harvested Kiwifruit. The rate of fruit respiration in both control and citral-treated fruits sharply increased in the first $15 \mathrm{~d}$ after treatment, followed by a sharp drop during the rest of storage periods (Figure 3(a)). The peak of respiration rate in the control kiwifruit was 1.56folds higher than that of the citral-treated kiwifruits. Respiration rate in the citral-treated kiwifruit was significantly $(p<0.05)$ lower than that in the controls during the entire storage periods (except for $75 \mathrm{~d}$ ).

Ripening index of the control and citral-treated kiwifruits increased over the period of cold storage (Figure 3(b)). Citral treatment delayed the increase of ripening index in kiwifruits. There was a lower level of ripening index in the citral-treated kiwifruit than that in the control fruits during storage period. The ripening index in the citral-treated kiwifruit was lower (12.7\%) than that of the control fruit at day 90.

The alterations of $\mathrm{O}_{2}{ }^{\bullet-}$ production rate in the control and citral-treated kiwifruit flesh were increased at the early storage ( $0 \mathrm{~d}$ to $45 \mathrm{~d})$ but afterwards declined (Figure 3(c)). Compared to the control fruits, the $\mathrm{O}_{2}{ }^{--}$production rate in the citral-treated kiwifruit retained lower levels during storage, with a significant $(p<0.05)$ difference during the middle and later periods of postharvest storage ( $45 \mathrm{~d}$ to $90 \mathrm{~d})$.

MDA is the final product in the peroxidation of membrane lipids, which can produce the Schiff alkali, destroying membrane integrity to promote membrane leakage. The MDA content in both control and citral-treated fruits increased with the prolongation of the storage time (Figure 3(d)). Citral treatment significantly $(p<0.05)$ delayed the accumulation of MDA content. The MDA content in the citral-treated kiwifruits was lower than that in the control fruits. There was a decrease (15.7\%) in the citraltreated kiwifruit at the end of storage period. It is worth mentioning that there was a prominent difference $(p<0.05$ or $p<0.01)$ between the control and citral-treated kiwifruits during the entire storage period (Figure 3(d)). 


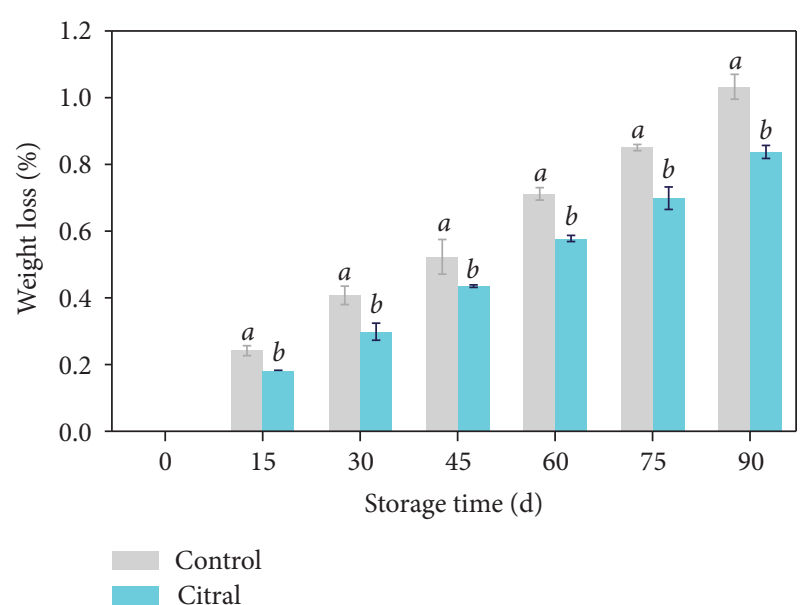

FIGURE 1: Effect of citral treatment on weight loss of harvested kiwifruit stored at $0-1^{\circ} \mathrm{C}$ for $90 \mathrm{~d}$. Vertical bars represent the mean \pm standard error (S.E., $n=3$ ). Letters indicate the statistical differences according to the independent samples $t$-test $(p<0.05)$ on each storage day.

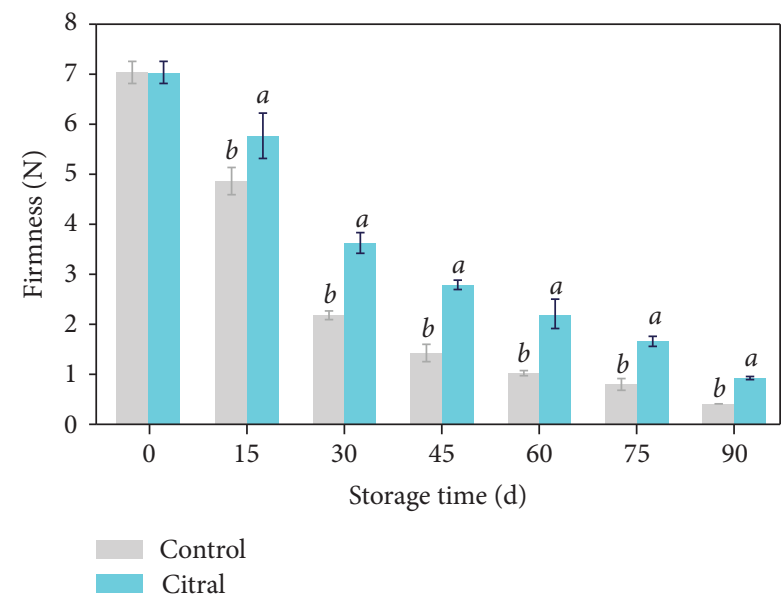

FIGURE 2: Effect of citral treatment on fresh firmness of harvested kiwifruit stored at $0-1^{\circ} \mathrm{C}$ for $90 \mathrm{~d}$. Vertical bars represent the mean \pm standard error (S.E., $n=3$ ). Letters indicate the statistical differences according to the independent samples $t$-test $(p<0.05)$ on each storage day.

\subsection{Effects of Citral Treatment on Antioxidant Capacity of} Harvested Kiwifruit. The AsA content in the flesh decreased gradually as storage time prolonged in both control and citral-treated kiwifruits (Figure 4(a)), with the citral-treated fruit exhibiting a slower decrease of AsA content. Statistical analysis showed that AsA content in the citral-treated fruits was significantly $(p<0.05)$ higher than that of the control fruits throughout the cold storage period.

By increasing in storage time, the TPC was reduced in both control and citral-treated kiwifruits. However, this decline of TPC was less prominent in kiwifruits subjected to the postharvest application of citral (Figure 4(b)). Overall results suggested that citral treatment exerted a significant inhibition on the degradation of TFC compared to its control fruits after $30 \mathrm{~d}$ of storage.
The TFC depicted the same trend as TPC, where the former in both control and citral-treated kiwifruits decreased over the period of cold storage (Figure 4(c)). Citral treatment prevented the decline of TFC in kiwifruit flesh, which maintained higher level of TFC in comparison with the control sample (Figure 4(c)). The TFC in the citraltreated fruits was significantly $(p<0.05)$ higher than that of the control fruits during the whole of storage period.

The DPPH scavenging capacity was decreased gradually during storage time in both control and citral-treated fruits (Figure 4(d)). Citral treatment significantly $(p<0.05)$ inhibited the decrease of DPPH scavenging capacity during the middle and later period of storage $(45 \mathrm{~d}$ to $90 \mathrm{~d})$. Kiwifruits treated with citral exhibited a remarkable higher levels of DPPH scavenging capacity than the control fruits.

The FRAP showed a similar pattern with DPPH scavenging capacity in both control and citral-treated fruits during storage time (Figure 4(e)). In addition, the FRAP in the citral-treated fruits was significantly higher $(p<0.05)$ than that in the control fruits. Moreover, there was a prominent difference between the control and citral-treated kiwifruits during the entire storage period (except for $60 \mathrm{~d}$ ).

3.4. Effects of Citral Treatment on SOD, CAT, and POD Activities of Harvested Kiwifruit. Antioxidant enzymes such as SOD, CAT, and POD serve an indispensable role in scavenging ROS in horticultural fruits. Both SOD and CAT activities exhibited similar tendency; i.e., they gradually increased during the initial storage of $45 \mathrm{~d}$ and then dropped rapidly. The activities of SOD and CAT in the citral-treated fruits were consistently higher $(p<0.05)$ than those in the control fruits, and there was a prominent difference between the control and citral-treated kiwifruits during the entire storage period (Figures 5(a) and 5(b)). The POD activity in both control and citral-treated fruits increased for $0-75 \mathrm{~d}$ and then declined in the following storage period. The POD activity significantly increased by citral treatment after treatment, and even at $75 \mathrm{~d}$ the POD levels still remained 1.15 times higher than those in the control fruits (Figure 5(c)). It is worth mentioning that an obvious significance was detected between the control and citral-treated groups during the whole storage period up to $90 \mathrm{~d}$ (except for $45 \mathrm{~d})$.

\section{Discussion}

The application of plant essential oils could effectively control postharvest fruit diseases by enhancing the antioxidant ability of many horticultural products that have extensively been reported recently [29, 30]. It is widely accepted that the important fruit quality parameters evaluating kiwifruit acceptability by consumers include fruit weight, firmness, color, and fragrance $[31,32]$. However, fruit quality of kiwifruit declined rapidly in both storage period and shelf life. Consequently, help to develop an effective preservations method for maintaining postharvest fruit quality of kiwifruits is of great interest $[6,32]$. However, there is hardly any study that describes the effect of citral 


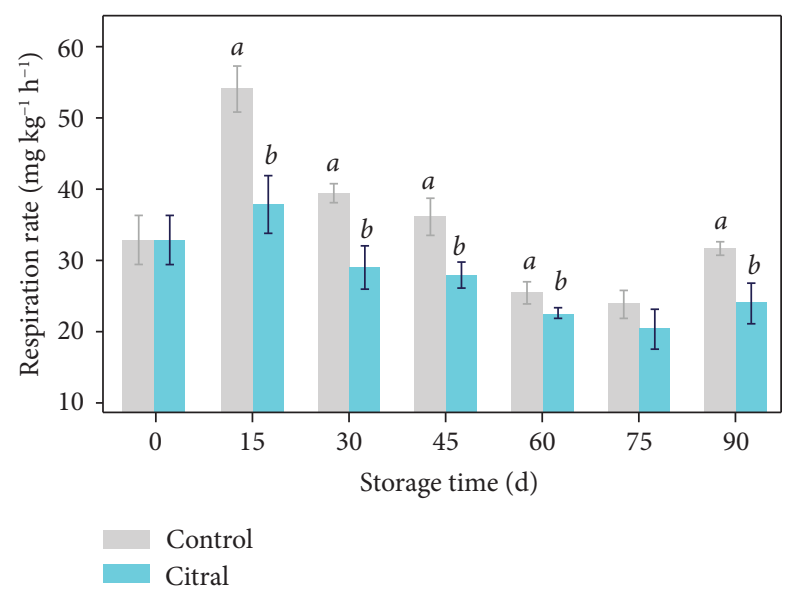

(a)

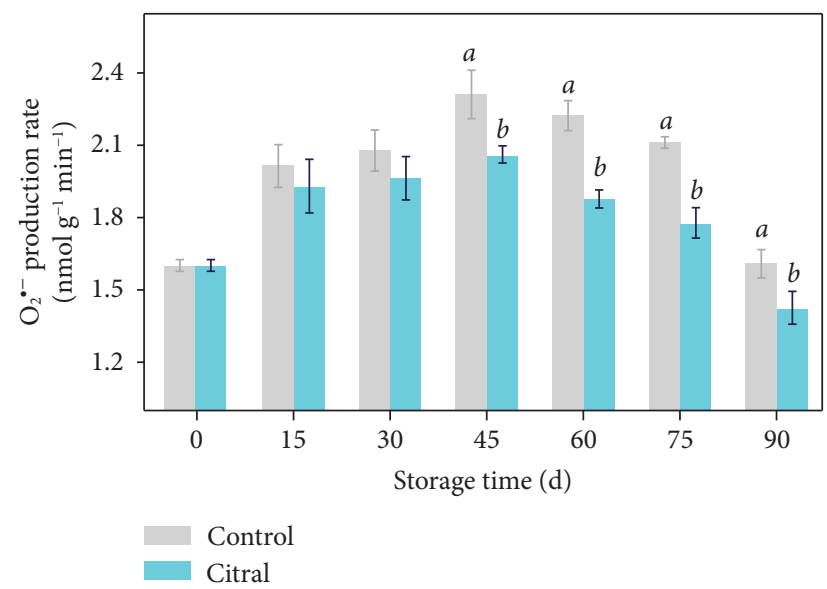

(c)

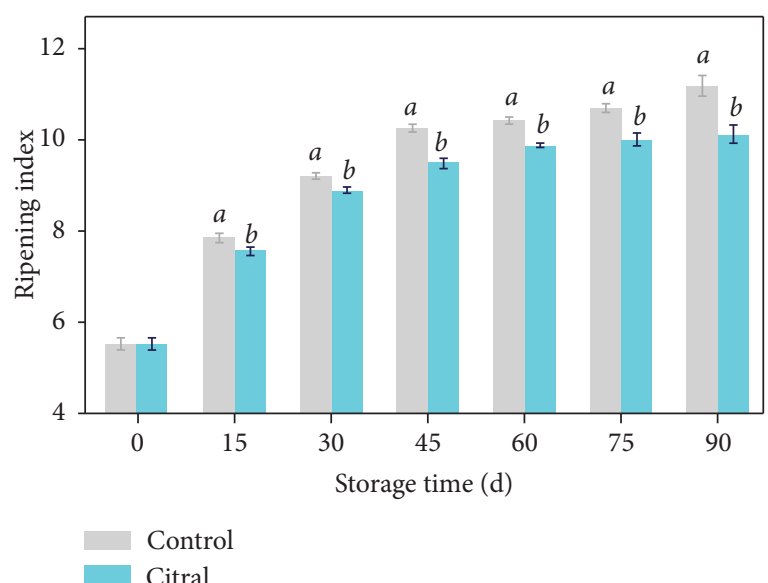

(b)

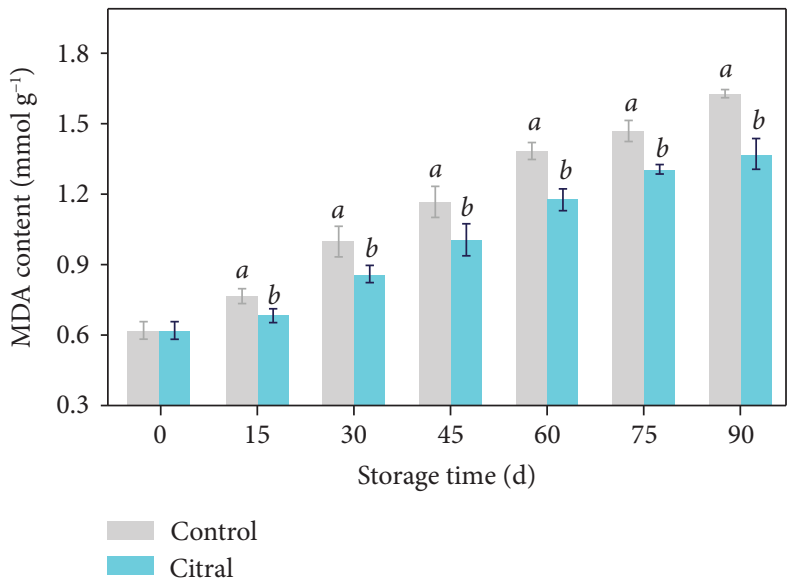

(d)

FIgURE 3: Effect of citral treatment on respiration rate, (a) ripening index, (b) $\mathrm{O}_{2}{ }^{--}$production rate, (c) and MDA content (d) of harvested kiwifruit stored at $0-1^{\circ} \mathrm{C}$ for $90 \mathrm{~d}$. Vertical bars represent the mean \pm standard error (S.E., $n=3$ ). Letters indicate the statistical differences according to the independent samples $t$-test $(p<0.05)$ on each storage day.

treatment on kiwifruit postharvest quality during cold storage or analyzes different quality parameters related to senescence stress. The current study examined that kiwifruits treated with citral exhibited lower ripening index while maintaining the antioxidant capacity in comparison with the control fruits.

For the senescence related parameters, citral treatment reduced changes on weight loss, firmness, respiration rate, and ripening index throughout the storage period of kiwifruits. In the current study, an increase in ripening index showed the process of kiwifruit ripening and senescence after harvest, which was significantly delayed in the citraltreated kiwifruits (Figure 3). A similar response has been reported following $5 \mu \mathrm{mol} \mathrm{L}^{-1} 24$-epibrassinolide treatment during storage in kiwifruit, by Wang et al. [6].

Ascorbic acid (AsA) could protect kiwifruits from the oxidative damage due to higher scavenging capacity for various ROS through the ascorbate peroxidase reaction [26]. A huge loss of AsA content occurred in the control treatment followed by citral treatment quite similar to a recent study where citral was applied during postharvest storage that declined AsA content in tomato during cold storage [24]. The results in the present study indicated that citral treatment could delay the oxidation process in kiwifruits by maintaining higher levels of AsA content than the control fruits after the harvest.

Both phenolics and flavonoids compounds are effective antioxidants that contribute to eliminating ROS. The current study revealed that citral treatment maintained the higher levels of both TPC and TFC in comparison with control kiwifruits. This is further accompanied with higher levels of DPPH scavenging capacity and FRAP, thereby reducing the damage caused by the ROS. These results indicated that both TPC and TFC were positively correlated with DPPH scavenging capacity and FRAP and negatively correlated with the ROS damage in kiwifruits. Several studies have also reported the inalienable relationship between phenolics content and their antioxidant capacity in postharvest fruits. Jin et al. [33] demonstrated that higher antioxidant capacity in Chinese bayberries treated with linalool and cinnamaldehyde was mainly linked to the higher phenolics contents. The high levels of antioxidant contents may delay 


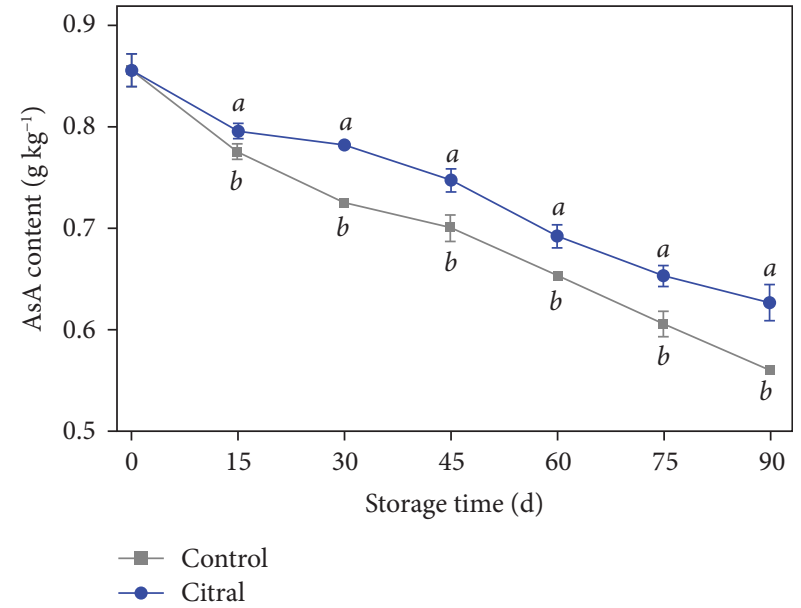

(a)

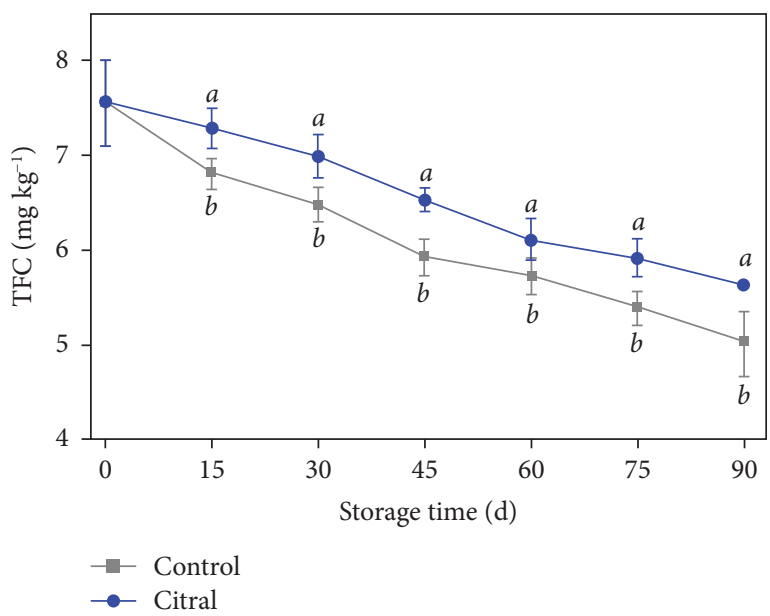

(c)

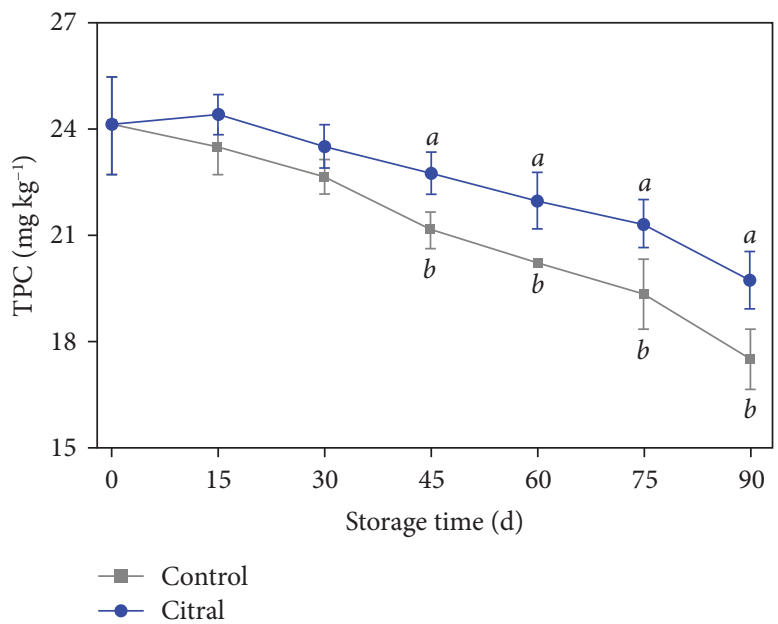

(b)

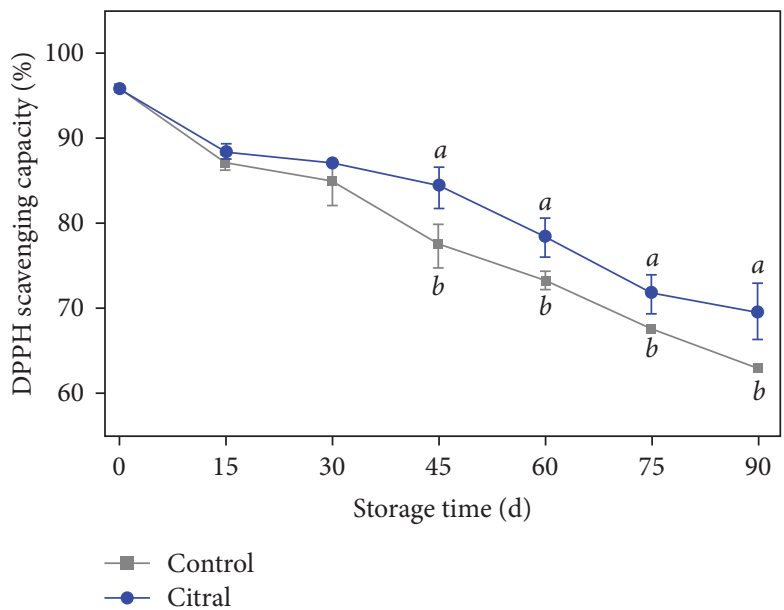

(d)

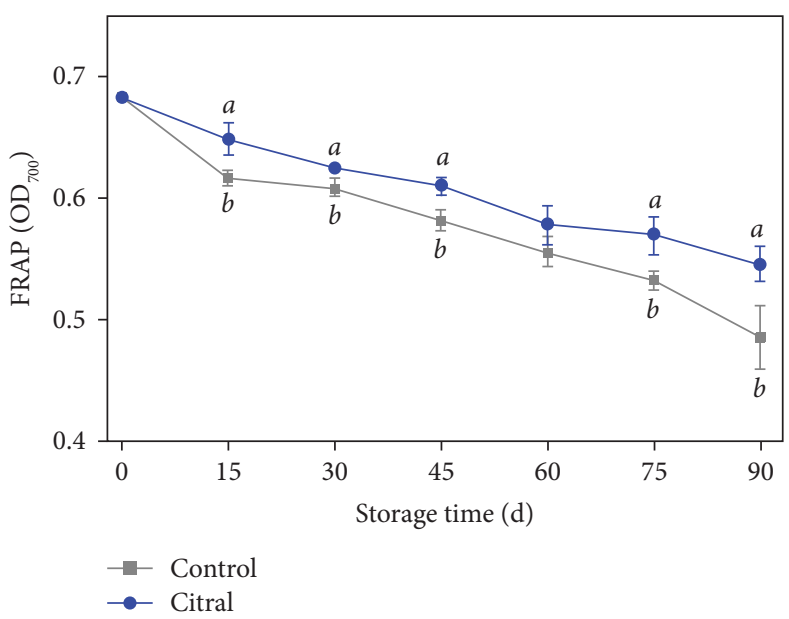

(e)

Figure 4: Effect of citral treatment on AsA content, (a) total phenolics content (TPC), (b) total flavonoids content (TFC), (c) 2,2-diphenyl-1picrylhydrazyl (DPPH) scavenging capacity, (d) and ferric reducing antioxidant power (FRAP) (e) of harvested kiwifruit stored at $0-1^{\circ} \mathrm{C}$ for $90 \mathrm{~d}$. Vertical bars represent the mean \pm standard error (S.E., $n=3$ ). Letters indicate the statistical differences according to the independent samples $t$-test $(p<0.05)$ on each storage day. 


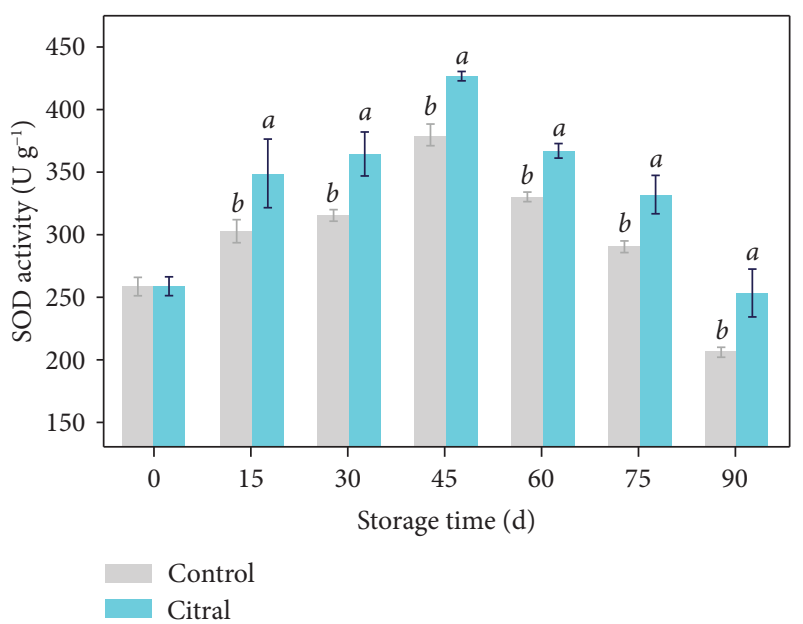

(a)

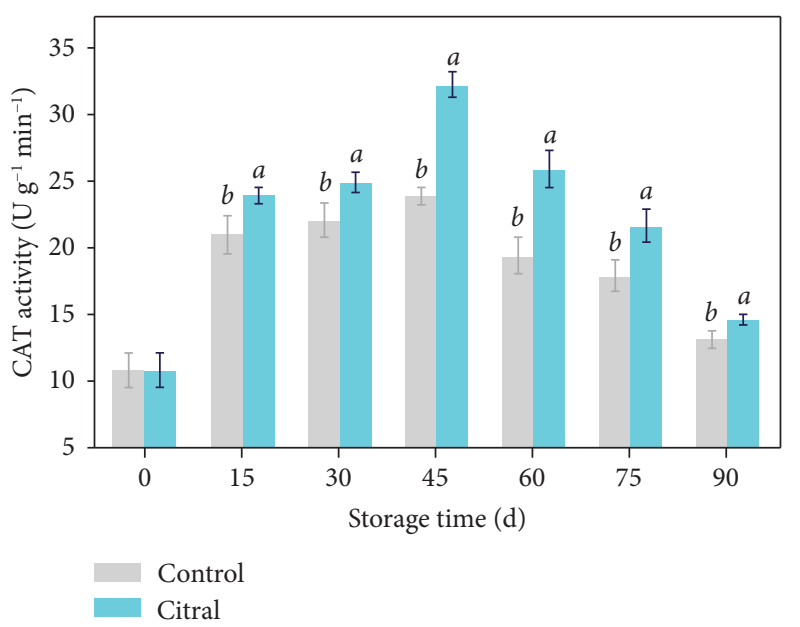

(b)

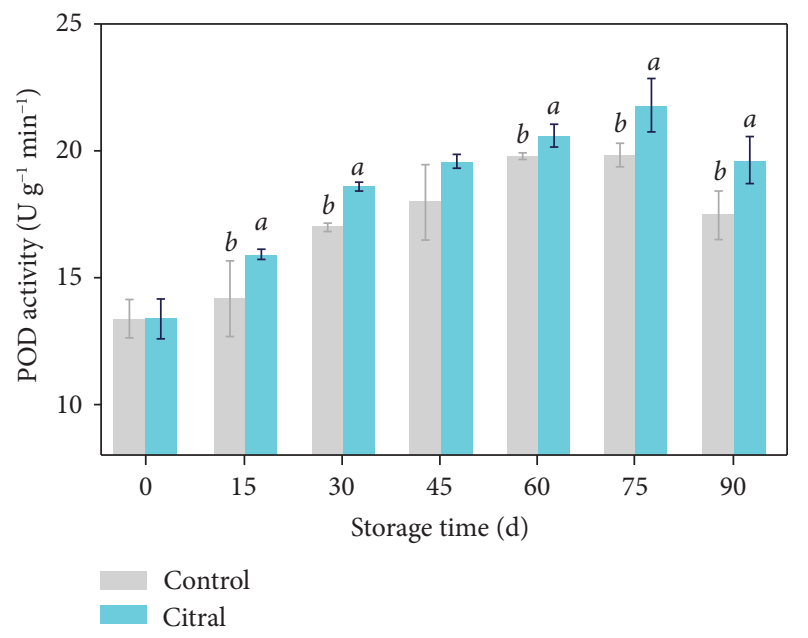

(c)

FIgURE 5: Effect of citral treatment on the activities of superoxide dismutase (SOD), (a) catalase (CAT), (b) and peroxidase (POD) (c) of harvested kiwifruit stored at $0-1^{\circ} \mathrm{C}$ for $90 \mathrm{~d}$. Vertical bars represent the mean \pm standard error (S.E., $\left.n=3\right)$. Letters indicate the statistical differences according to the independent samples $t$-test $(p<0.05)$ on each storage day.

the physiological deterioration and hence postpone the fruit senescence.

Respiration rate is an important parameter for postharvest fruit metabolic activity and quality assessment. The findings herein showed that citral-treated fruits exhibit lower respiration rates and hence offer better storage quality. In addition, MDA content is another indicator for cellular oxidative damages. Lipid peroxidation is a major reason for membrane integrity damages during fruits senescence [34]. High MDA content could cause the damage in cellular membrane systems, which further led to the softening of kiwifruits. The current results indicated that citral treatment could reduce the MDA accumulation in kiwifruits to resist the senescence induced ROS damage, thereby delaying postharvest senescence in kiwifruits. Furthermore, similar findings in kiwifruits have been observed when treated with 24-epibrassinolide [6].

The ripening process in plant has been seen as an oxidative phenomenon accompanied by burdens of $\mathrm{ROS}\left(\mathrm{H}_{2} \mathrm{O}_{2}\right.$ and $\mathrm{O}_{2}{ }^{--}$) [35]. The ROS burst may result in oxidative stress mediated damages to the plant tissue specifically the membranes. Herein, the citral-treated kiwifruits had significantly lower level of $\mathrm{O}_{2}{ }^{--}$production rate, which suggested that citral treatment was better for protecting kiwifruit against the ROS-mediated oxidative damages. The elimination of ROS is linked with the activities of antioxidant enzymes such as SOD, CAT, and POD in fruits and vegetables $[6,13,36]$. SOD plays a key role in the dismutation of $\mathrm{O}_{2}{ }^{--}$into $\mathrm{H}_{2} \mathrm{O}_{2}$, while $\mathrm{H}_{2} \mathrm{O}_{2}$ is obliterated by CAT which is indispensable for the detoxification of ROS [11]. POD is the key enzyme in the biosynthesis and oxidation of phenolics compounds [37]. Therefore, higher antioxidant enzyme activities and their coordinated actions might be the underlying mechanism for reducing lipid peroxidation and delaying senescence in horticultural fruits $[35,36]$. The exogenous treatment of methyl jasmonate (MeJA) help induce the increased activities of POD and SOD, thereby reducing oxidative damage and enhancing disease resistance in kiwifruit [3]. 
Meanwhile, alginate oligosaccharide (AOS) treatment elevated SOD and CAT activities, which contributed to enhancing abiotic stress resistance and delaying postharvest senescence in kiwifruits [2]. Herein, citral treatment induces higher levels of SOD, CAT, and POD activities compared with the respective controls. Thus, the findings suggested that citral treatment effectively induces the increases in antioxidant enzymes activities and helps coordinate the balance of ROS level in kiwifruits. The link between these antioxidant enzymes might be the major factor that triggers the oxidation resistance, thereby maintaining postharvest quality and prolonging storage time of kiwifruits.

\section{Conclusions}

The beneficial effects of citral treatment on postharvest quality and antioxidant capacity of 'Jinkui' kiwifruit were investigated in the present study. The study revealed that citral treatment has significantly decreased fruit weight loss, delayed softening, alleviated senescence progress, and maintained higher levels of antioxidant contents that help boost antioxidant enzyme activities in comparison with the respective control fruits during storage. The alleviation of postharvest senescence in kiwifruits treated with citral might be attributed to maintaining antioxidant capacity, higher levels of AsA content, TPC, and TFC, contributing to enhanced DPPH scavenging capacity and FRAP. Furthermore, weight loss, respiration rate, and ripening index were lower in the citral-treated kiwifruits than those in the control fruits, which have been dedicated to less consumptions of nutrients and have got extended storage time. Therefore, it is concluded that citral treatment could serve as a potential and feasible preservation method for delaying postharvest senescence decay and maintaining antioxidant capacity in 'Jinkui' kiwifruit.

\section{Data Availability}

All the statistical data used to support the findings of this study are included within the paper.

\section{Conflicts of Interest}

The authors declare no conflicts of interest.

\section{Acknowledgments}

This research was supported by the National Natural Science Foundation of China (31760598 and 32060703), the Natural Science Foundation and Advantage Innovation Team Project of Jiangxi Province (20171BCB24006 and 20181BCB24005), and Jiangxi Provincial Department of Education Research Project (GJJ190241).

\section{References}

[1] M. Guldas, "The determination of ascorbic acid, chlorophyll and pectin contents of Turkish kiwifruit," Journal of Food Quality, vol. 26, no. 4, pp. 353-358, 2003.
[2] J. Liu, J. F. Kennedy, X. Zhang et al., "Preparation of alginate oligosaccharide and its effects on decay control and quality maintenance of harvested kiwifruit," Carbohydrate Polymers, vol. 242, Article ID 116462, 2020.

[3] L. Pan, X. Zhao, M. Chen, Y. Fu, M. Xiang, and J. Chen, "Effect of exogenous methyl jasmonate treatment on disease resistance of postharvest kiwifruit," Food Chemistry, vol. 305, Article ID 125483, 2020.

[4] F. Zheng, W. Zheng, L. Li et al., "Chitosan controls postharvest decay and elicits defense response in kiwifruit," Food and Bioprocess Technology, vol. 10, no. 11, pp. 1937-1945, 2017.

[5] H. Hu, H. Zhou, and P. Li, "Lacquer wax coating improves the sensory and quality attributes of kiwifruit during ambient storage," Scientia Horticulturae, vol. 244, pp. 31-41, 2019.

[6] X. Wang, Z. Lu, J. Su, Y. Li, M. Cao, and H. Gao, "24-epibrassinolide delays senescence in harvested kiwifruit through effects on mitochondrial membrane and antioxidant activity," Lwt, vol. 118, Article ID 108833, 2020.

[7] Y. Zhu, J. Yu, J. K. Brecht, T. Jiang, and X. Zheng, "Pre-harvest application of oxalic acid increases quality and resistance to Penicillium expansum in kiwifruit during postharvest storage," Food Chemistry, vol. 190, pp. 537-543, 2016.

[8] M. Ali, M.-M. Liu, Z.-E. Wang, S.-E. Li, T.-J. Jiang, and X.-L. Zheng, "Pre-harvest spraying of oxalic acid improves postharvest quality associated with increase in ascorbic acid and regulation of ethanol fermentation in kiwifruit cv. Bruno during storage," Journal of Integrative Agriculture, vol. 18, no. 11, pp. 2514-2520, 2019.

[9] B. Ozturk, S. Uzun, and O. Karakaya, "Combined effects of aminoethoxyvinylglycine and map on the fruit quality of kiwifruit during cold storage and shelf life," Scientia Horticulturae, vol. 251, pp. 209-214, 2019.

[10] B. Öztürk and F. Yücedağ, "Effects of methyl jasmonate on quality properties and phytochemical compounds of kiwifruit (actinidia deliciosa cv. 'Hayward') during cold storage and shelf life," Turkish Journal of Agriculture and Forestry, vol. 45, 2021, in press.

[11] L. Zhu, W. Wang, J. Shi et al., "Hydrogen sulfide extends the postharvest life and enhances antioxidant activity of kiwifruit during storage," Journal of the Science of Food and Agriculture, vol. 94, no. 13, pp. 2699-2704, 2014.

[12] X. Lin, R. Yang, Y. Dou et al., "Transcriptome analysis reveals delaying of the ripening and cell-wall degradation of kiwifruit by hydrogen sulfide," Journal of the Science of Food and Agriculture, vol. 100, no. 5, pp. 2280-2287, 2020.

[13] V. Goffi, A. Magri, R. Botondi, and M. Petriccione, "Response of antioxidant system to postharvest ozone treatment in "Soreli" kiwifruit," Journal of the Science of Food and Agriculture, vol. 100, no. 3, pp. 961-968, 2019.

[14] B. Prakash, P. K. Mishra, A. Kedia, A. K. Dwivedy, and N. K. Dubey, "Efficacy of some essential oil components as food preservatives against food contaminating molds, aflatoxin B1 production and free radical generation," Journal of Food Quality, vol. 38, no. 4, pp. 231-239, 2015.

[15] B. A. Bahri, G. Mechichi, W. Rouissi, I. Ben Haj Jilani, and Z. Ghrabi-Gammar, "Effects of cold-storage facility characteristics on the virulence and sporulation of Penicillium expansum and the efficacy of essential oils against blue mold rot of apples," Folia Horticulturae, vol. 31, no. 2, pp. 301-317, 2019.

[16] J. He, D. Wu, Q. Zhang et al., "Efficacy and mechanism of cinnamon essential oil on inhibition of Colletotrichum acutatum isolated from 'Hongyang' kiwifruit," Frontiers in Microbiology, vol. 9, 2018. 
[17] F. Wu and X. D. Zheng, "Essential oils to control Alternaria alternata in vitro and in vivo," Food Control, vol. 18, pp. 1126-1130, 2007.

[18] C. Y. Wang, S. Y. Wang, J.-J. Yin, J. Parry, and L. L. Yu, "Enhancing antioxidant, antiproliferation, and free radical scavenging activities in strawberries with essential oils," Journal of Agricultural and Food Chemistry, vol. 55, no. 16, pp. 6527-6532, 2007.

[19] C. Y. Wang, S. Y. Wang, and C. Chen, "Increasing antioxidant activity and reducing decay of blueberries by essential oils," Journal of Agricultural and Food Chemistry, vol. 56, no. 10, p. $3587,2008$.

[20] M. Maswal and A. A. Dar, "Formulation challenges in encapsulation and delivery of citral for improved food quality," Food Hydrocolloids, vol. 37, pp. 182-195, 2013.

[21] E. Wuryatmo, A. J. Able, C. M. Ford, and E. S. Scott, "Effect of volatile citral on the development of blue mould, green mould and sour rot on navel orange," Australasian Plant Pathology, vol. 43, no. 4, pp. 403-411, 2014.

[22] H. Zhou, N. Tao, and L. Jia, "Antifungal activity of citral, octanal and $\alpha$-terpineol against Geotrichum citri-aurantii," Food Control, vol. 37, pp. 277-283, 2014.

[23] Y. Wang, T. Shan, Y. Yuan, and T. Yue, "Overall quality properties of kiwifruit treated by cinnamaldehyde and citral: microbial, antioxidant capacity during cold storage," Journal of Food Science, vol. 81, no. 12, Article ID H3043, H3051 pages, 2016.

[24] S. L. Du, J. H. Zhang, S. Y. Chen, S. Ma, and L. Zeng, "The combined effect of 1-methylcyclopropene and citral suppressed postharvest grey mould of tomato fruit by inhibiting the growth of Botrytis cinerea," Journal of Phytopathology, vol. 167, pp. 123-134, 2019.

[25] M. Horák, P. Šnurkovič, I. Ondrášek, J. Balík, and V. Srilaong, "Comparison of some physico-chemical parameters of kiwiberry (Actinidia arguta) cultivars from a cold climate," Folia Horticulturae, vol. 31, no. 2, pp. 375-383, 2019.

[26] Z. Nie, Q. Huang, C. Chen, C. Wan, and J. Chen, "Chitosan coating alleviates postharvest juice sac granulation by mitigating ros accumulation in harvested pummelo (Citrus grandis L. Osbeck) during room temperature storage," Postharvest Biology and Technology, vol. 169, Article ID 111309, 2020.

[27] H. Jiang, Z. Sun, R. Jia, X. Wang, and J. Huang, "Effect of chitosan as an antifungal and preservative agent on postharvest blueberry," Journal of Food Quality, vol. 39, no. 5, pp. 516-523, 2016.

[28] I. F. F. Benzie and J. J. Strain, "The ferric reducing ability of plasma (FRAP) as a measure of antioxidant power: the FRAP assay," Analytical Biochemistry, vol. 239, no. 1, pp. 70-76, 1996.

[29] J. M. Valverde, M. J. Giménez, F. Guillén, D. Valero, D. Martínez-Romero, and M. Serrano, "Methyl salicylate treatments of sweet cherry trees increase antioxidant systems in fruit at harvest and during storage," Postharvest Biology and Technology, vol. 109, pp. 106-113, 2015.

[30] C. Chen, N. Cai, J. Chen, and C. Wan, "Clove essential oil as an alternative approach to control postharvest blue mold caused by Penicillium italicum in citrus fruit," Biomolecules, vol. 9, no. 5, p. 197, 2019.

[31] H. Huang, L. Guo, L. Wang et al., "1-methylcyclopropene (1MCP) slows ripening of kiwifruit and affects energy status, membrane fatty acid contents and cell membrane integrity," Postharvest Biology and Technology, vol. 156, Article ID 110941, 2019.
[32] Y. J. Lim and S. H. Eom, "Kiwifruit cultivar "Halla gold" functional component changes during preharvest fruit maturation and postharvest storage," Scientia Horticulturae, vol. 234, pp. 134-139, 2018.

[33] P. Jin, X. Wu, F. Xu, X. Wang, J. Wang, and Y. Zheng, "Enhancing antioxidant capacity and reducing decay of Chinese bayberries by essential oils," Journal of Agricultural and Food Chemistry, vol. 60, no. 14, pp. 3769-3775, 2012.

[34] Q. Hu, Y. Fang, Y. Yang, N. Ma, and L. Zhao, "Effect of nanocomposite-based packaging on postharvest quality of ethylene-treated kiwifruit (Actinidia deliciosa) during cold storage," Food Research International, vol. 44, no. 6, pp. 1589-1596, 2011.

[35] Y. Xia, T. Chen, G. Qin, B. Li, and S. Tian, "Synergistic action of antioxidative systems contributes to the alleviation of senescence in kiwifruit," Postharvest Biology and Technology, vol. 111, pp. 15-24, 2016.

[36] S. S. Gill and N. Tuteja, "Reactive oxygen species and antioxidant machinery in abiotic stress tolerance in crop plants," Plant Physiology and Biochemistry, vol. 48, no. 12, pp. 909930, 2010.

[37] C. H. Foyer, P. Descourvieres, and K. J. Kunert, "Protection against oxygen radicals: an important defence mechanism studied in transgenic plants," Plant, Cell and Environment, vol. 17, no. 5, pp. 507-523, 1994. 